\title{
Unsupervised Multi-View CNN for Salient View Selection of 3D Objects and Scenes
}

Paper ID 3254

Abstract. We present an unsupervised 3D deep learning framework based on a ubiquitously true proposition named by us view-object consistency as it states that a $3 \mathrm{D}$ object and its projected 2D views always belong to the same object class. To validate its effectiveness, we design a multi-view CNN instantiating it for the salient view selection of 3D objects, which quintessentially cannot be handled by supervised learning due to the difficulty of collecting sufficient and consistent training data. Our unsupervised multi-view CNN branches off two channels which encode the knowledge within each $2 \mathrm{D}$ view and the 3D object respectively and also exploits both intra-view and inter-view knowledge of the object. It ends with a new loss layer which formulates the view-object consistency by impelling the two channels to generate consistent classification outcomes. We evaluate our method both qualitatively and quantitatively, demonstrating its superiority over several state-of-the-art methods. In addition, we showcase that our method can be used to select salient views of $3 \mathrm{D}$ scenes containing multiple objects which is a more challenging and less investigated problem.

Keywords: Unsupervised 3D Deep Learning, Multi-View CNN, View-Object Consistency, View Selection

\section{Introduction}

The success of Generative Adversarial Network (GAN) [8] that introduced the adversarial idea has demonstrated the great value and impact of unsupervised deep learning framework that is a widely applicable. One important reason is that the manual collection and annotation of a large dataset for training a deep neural network is laborious in many tasks. This is particularly the case for 3D tasks where data collection and annotation are generally more challenging than those in 2D tasks. Therefore, a widely applicable 3D deep learning framework is potentially of broad interest in the fields of $3 \mathrm{D}$ vision, computer graphics and machine learning.

A simple but ubiquitously true proposition is that a $3 \mathrm{D}$ object and its projected $2 \mathrm{D}$ views always belong to the same object class no matter what taxonomy is applied to the classification. We name the proposition view-object consistency and propose a novel unsupervised 3D deep learning framework based on it. Since it is not feasible for us to solidly and thoroughly explore the utility of the framework through various 3D tasks in one paper, we intentionally pick the task of salient view selection of 3D objects to demonstrate its effectiveness for three reasons. First, salient view selection of $3 \mathrm{D}$ objects is challenging as it does not only rely on low-level geometric features 
but also involves complex high-level semantic understandings of the objects. Thus a data-driven method is naturally sound. Second, however, it is the particular task where collecting a large amount of accurately and consistently annotated data is notoriously difficult. We found that all existing datasets are very small (e.g. 68 objects in [7] and 16 objects in [24]) no matter whether the annotations were collected directly (e.g. by asking human subjects to mark a viewpoint on a view sphere surrounding the object [7]) or indirectly (e.g. by paired comparisons where subjects were asked to select the preferred view from two views for multiple times [24]). Third, we shall further show the advantage of an unsupervised method by extending salient view selection to 3D scenes which is more challenging and less investigated. Salient view selection of 3D scenes can hardly be addressed by a weakly supervised method relying on such annotation as a single class label because a scene contains objects belonging to different classes.

The problem of salient view selection of $3 \mathrm{D}$ objects is arguably well defined. Besides the chunk of related literatures in computer vision and graphics that will be discussed in Section 2, researchers in psychology [6,2] have revealed that for many classes of familiar objects, the preferred views are reasonably consistent among the human subjects. To make it clear, the most salient view of a 3D object herein is defined as the view that a human subject likes most for whatever reason. And we shall evaluate our method using the publicly available benchmark [7] where subjects were asked to rotate a 3D object to directly select the view that they preferred.

To instantiate the proposition of view-object consistency in the context of both 3D deep learning and salient view selection, we develop a new multi-view convolutional neural network (CNN). It formulates the view-object consistency through a two-channel deep architecture and a newly designed loss function. It also integrates the neural network with an important heuristic of human's view preference through a specifically designed layer. The proposed multi-view CNN is trained end-to-end in an unsupervised manner using only a collection of 3D objects without any manual annotations and is thus named as UMVCNN, short for Unsupervised Multi-View CNN. As a whole, it effectively exploits both intra-view and inter-view knowledge via a multi-view representation of 3D objects for salient view selection.

The contribution of our work is hence threefold:

(1) We propose a novel unsupervised framework of 3D deep learning where the core idea is valid ubiquitously and thus potentially has a wide range of applications.

(2) We propose a new multi-view CNN in accordance with this unsupervised framework to address the classical problem of salient view selection of 3D objects .

(3) With the aid of the unsupervised 3D deep learning framework, we extend salient view selection from individual 3D objects to scenes containing multiple 3D objects.

\section{Related work}

We categorise previous work on view selection of 3D objects into three groups. The first group of methods are based only on handcrafted attributes of 3D objects; the second group is essentially shallow learning of a certain model to combine multiple attributes for view selection while all attributes are not learned but still handcrafted; the third group of methods are based on deep learning where some, if not all of the attributes, are learned via deep neural networks. 
Handcrafted attributes. Polonsky et al. [22] explored general frameworks for best view selection by analysing several handcrafted low-level attributes associated to the geometrical or statistical properties of the 3D object or its projected 2D views, including surface area entropy, visibility ratio, curvature entropy, silhouette length, silhouette entropy and topological complexity. Lee et al. [16] proposed to select the salient view of a 3D object using the attribute of mesh saliency computed based on Gaussian-weighted mean curvatures. Yamauchi et al. [34] also employed mesh saliency as the intra-view cue for finding the salient view while taking into account such inter-view cue as the similarity of the projected views. Lienhard et al. [18] used not only geometrical attributes but also esthetic and semantic views to find the good views for procedural 3D models. Leifman et al. [17] computed a saliency measure based on both local geometrical and global topological attributes to select salient views of 3D objects. However, in general, most methods based on handcrafted attributes do not generalise well due mainly to the limited expressive capabilities of the attributes extracted through some fixed schemes for objects of different classes.

Handcrafted attributes with shallow learning. Vieira et al. [30] learned good views complying with user preference via an SVM classifier where the candidate views were represented by a collection of handcrafted attributes. To investigate the view preference of humans, Secord et al. [24] collected a small dataset via a user study to learn a regression model which linearly combined a list of handcrafted attributes. Mezuman and Weiss [19] leveraged Internet image collections to learn the canonical view from which we most often see the object, where the handcrafted GIST descriptor was employed to measure view similarity. Zhao et al. [35] learned best views from hand-drawn sketches by asking participants to align a 3D model according to a given sketch. He et al. [10] proposed a multi-view learning framework exploiting both 2D and 3D handcrafted attributes to assess and recommend viewpoints for photographing architectures.

Deep learning. Apart from the psychological work [28, 6, 9], in computer vision, there is also evidence $[33,27,20]$ of the relation between view selection and object recognition where view-dependent attributes were extracted via deep neural networks for 3D object recognition. Kim et al. [14] and Song et al. [26] leveraged deep CNNs for salient view selection of objects instead of improving recognition accuracy. Our work is inspired by both of them but fundamentally different for two reasons: 1) both [14] and [26] require annotated data for training while our work is unsupervised with no need for data annotation; 2) both of them cannot be trained end-to-end where the former trains two CNNs and a Random Forest classifier separately and the latter trains a CNN and a Markov Random Field individually while our UMVCNN is trained fully end-to-end.

\section{Salient view selection via UMVCNN}

In this section, we first describe each component of our method in a piecewise manner. We then elaborate the implementation as a whole in both training and deployment modes where each component is situated in the context of the complete pipeline.

\subsection{Multi-view representation of a 3D object}

Multi-view CNNs have been widely used to adapt image-based deep networks to 3D objects where a $3 \mathrm{D}$ object is represented as a selection of its $2 \mathrm{D}$ projected views. Com- 


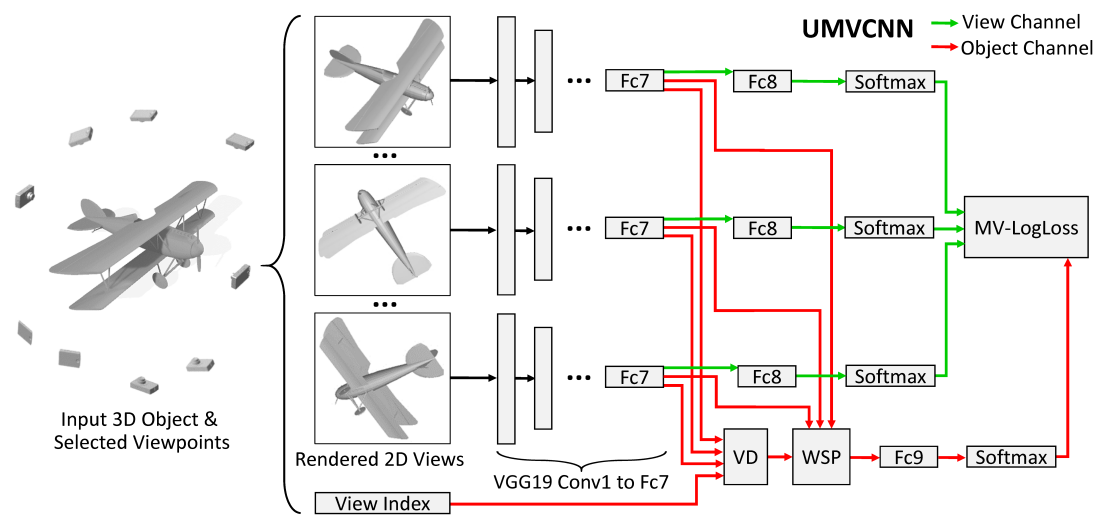

Fig. 1. Overview of the proposed UMVCNN containing two channels. The green and the red arrows denote the view channel and the object channel respectively. "VD" and "WSP" denote the view distinction and the weighted sum pooling layers respectively.

pared with other methods which try to generalise deep learning to non-Euclidean domains, multi-view CNNs showed state-of-the-art performance in various 3D shape understanding tasks $[27,23,13,12]$. One consensus among these tasks is that we should avoid using the very 'bad' views usually defined as the ones which potentially cause misrecognition or misunderstanding of the object. We propose a scheme which considers two low-level attributes to ensure that the selected $2 \mathrm{D}$ views for representing a $3 \mathrm{D}$ object are at least 'not very bad'.

We start with an icosahedron to uniformly sample a view sphere surrounding the input 3D object. Then we iteratively subdivide the icosahedron to produce more vertices (i.e., viewpoints) on the view sphere. We end with a polyhedron with 162 vertices. Next, we rank the views taken from these viewpoints based on the attributes of view area and silhouette length. View area is calculated as the area of the projection of the object as seen from a particular viewpoint. Silhouette length is the length of the outer contour of the silhouette of the object as seen from a particular viewpoint. We collect the top $N(N=20$ in this work) views with the highest ranks on average based on the two attributes as the multi-view representation of the $3 \mathrm{D}$ object.

\subsection{UMVCNN architecture}

Overview. Fig. 1 illustrates the architecture of the UMVCNN. It starts with the classic VGG-19 model [3] as the baseline architecture and then branches off the view channel and the object channel after the Fc7 layers. Through the newly designed view distinction (VD) layer, it generates an inter-view heuristic using the deep features extracted from the $2 \mathrm{D}$ views. A weighted sum pooling (WSP) layer is then employed to incorporate this heuristic and multiple intra-view features derived from each individual view into a single tensor encoding the information corresponding to the entire $3 \mathrm{D}$ object. These two layers and the newly added fully connected layer Fc9 followed by a Softmax normalisation form the object channel of the UMVCNN. It outputs to the loss layer a vector composed of the probabilities of the $3 \mathrm{D}$ object belonging to a certain class. On the other 
hand, we still keep the original Fc8 layer of VGG-19 in the view channel that generates a vector for each view predicting which class the view belongs to. Every VGG-19 layer from Conv1 to Fc8 in the UMVCNN shares the same weights for all views. Finally, the outputs of the view and the object channels converge at the newly designed MultiView Logistic Loss (MV-LogLoss) layer which formulates the view-object consistency to enable an unsupervised learning. Details are described in the following.

View distinction (VD) layer. Existing work [34, 24, 36] showed that human subjects find a good view by not only scrutinising its own intra-view content, but also comparing it with other views of the same object. Note that a limitation of most previous work is the lack of the consideration of such inter-view knowledge in their algorithms. In this work, we propose a heuristic mechanism to formulate the inter-view knowledge via paired comparisons of views. Previous work [32,15] in psychology pointed out that a basic principle in human visual system is to suppress the response to frequently occurring features, while at the same time it remains sensitive to features that deviate from the norm. We thus propose the VD layer as a heuristic method to formulate this principle where the view most different from all other views are regarded as the most distinct one. The VD layer takes as input the outputs of all Fc7 layers. Since one 3D object is represented as $N$ views, the input of the VD layer is a matrix of size $4096 \times N$ for a given object. Each of its columns can be regarded as a feature descriptor of one view. The VD layer outputs an $N$-dimensional vector to the WSP layer. Each element of the vector corresponds to the distinction of a particular view, reflecting how distinct that view is. The more distinct the view, the larger the contribution it will make in the aggregation of multi-view information implemented by the WSP layer.

Given two views $V_{i}$ and $V_{j}$, their difference can be measured as the Euclidean distance between their feature descriptors $F_{i}$ and $F_{j}$ output by the Fc7 layer (with ReLU activation). However, this measure is insufficient as a view tends to have similar content with its neighbouring views. If a view is even very different from its neighbouring views, it is likely to contain some unique content and thus be considered confidently distinct from the other views. Hence, the dissimilarity of two views should be proportional to the difference computed as the Euclidean distance between their feature descriptors and inversely proportional to the geodesic distance between their corresponding viewpoints on the view sphere. Such a heuristic also computationally holds for symmetric objects. For symmetric views, the dissimilarity is always 0 as $F_{i}=F_{j}$ and thus has nothing to do with the geodesic distance between them. Besides the $N$ projected views, the UMVCNN also requires as input the view index $\operatorname{VInd}_{i} \in\{1,2, \ldots, 162\}$ generated as a byproduct when creating the multi-view presentation of the object (see Section 3.1).

Let $\operatorname{Geod}\left(\mathrm{VInd}_{i}, \mathrm{VInd}_{j}\right)$ be the geodesic distance between the viewpoints corresponding to $V_{i}$ and $V_{j}$, the dissimilarity between the two views is defined as:

$$
D_{i j}=\frac{\left\|F_{i}-F_{j}\right\|}{\left.1+\alpha \cdot \operatorname{Geod}\left(\operatorname{VInd}_{i}, \operatorname{VInd}_{j}\right)\right)}, \quad \text { s.t. } i, j \in\{1,2 \ldots, N\} \text { and } i \neq j
$$

where $\alpha=2$ in our implementation. The distinction of $V_{i}$ is then computed as the sum of its pairwise dissimilarity to all the other views.

$$
S_{i}=\sum_{j \neq i} D_{i j}
$$


Both Eq. (1) and Eq. (2) are differentiable. So for back-propagation, given that the gradient passed to the VD layer is an $N$-dimensional vector $\mathcal{S}$, according to the chain rule, the gradient $\mathcal{F}$ of this layer with regard to its input can be computed as

$$
\mathcal{F}_{i}=\mathcal{S}_{i} \frac{\partial S_{i}}{\partial F_{i}}
$$

Considering Eqs. (1) and (2) and the partial derivative of the Euclidean distance function $\frac{\partial\|x\|}{\partial x_{i}}=\frac{x_{i}}{\|x\|}$, it can be computed as

$$
\frac{\partial S_{i}}{\partial F_{i}}=\sum_{j \neq i} \frac{F_{i}-F_{j}}{\left(1+\alpha \cdot \operatorname{Geod}\left(\operatorname{VInd}_{i}, \operatorname{VInd}_{j}\right)\right) \cdot\left\|F_{i}-F_{j}\right\|} .
$$

Weighted sum pooling (WSP) layer. To implement the view-object consistency through the loss layer which requires that the outputs of the view and the object channels have the same dimensions, we need to pool to aggregate the learned knowledge across all the $2 \mathrm{D}$ views to create a single descriptor for the $3 \mathrm{D}$ object. Also, we need to consider how to cast the influence of view distinction into this aggregation process where distinct views should have larger weights. Thus instead of the popular elementwise max pooling $[27,13]$ in multi-view CNNs, we carry out a WSP to incorporate view distinction as the weights into the pooling

$$
P=\sum_{i=1}^{N} F_{i} S_{i}
$$

where $F_{i}$ is the column vector of the output of the Fc7 layer $F$ which denotes the feature descriptor of view $V_{i}$ and $S_{i}$ is its distinction output by the VD layer. It shows that the output of the WSP layer $P$ regarded as the feature descriptor of the $3 \mathrm{D}$ object is estimated as the weighted sum of the feature descriptors of all the views where the weights are their distinctions. Eq. (5) can be expressed in a bilinear form as $P=F S$. Thus in the back-propagation, the gradients $\mathcal{F}$ and $\mathcal{S}$ of the WSP layer with regard to its inputs $F$ and $S$ respectively can be computed as

$$
\mathcal{F}=\mathcal{P} S^{T}, \quad \mathcal{S}=F^{T} \mathcal{P}
$$

where $\mathcal{P}$ denotes the gradient passed to the WSP layer.

MV-LogLoss Layer. We proposed the MV-LogLoss layer to formulate the proposition of view-object consistency, which enables an unsupervised learning. The basic idea herein is that no matter what the taxonomy is, the outcome of the classification based on the information of each $2 \mathrm{D}$ view should be consistent with that based on the entire 3D object. Note that as illustrated in Fig. 1, either of the view and the object channels alone is specifically designed to have the architecture of a classification network, which significantly facilitates the formulation of the view-object consistency. Moreover, such a design benefits salient view selection as the features vital for object classification are usually also important for the selection of a salient view. Psychological studies [28, 6, 9] have validated the strong correlation between view selection and object recognition: a good view of an object can significantly help people to correctly recognise it. 
The MV-LogLoss simply adapts the log loss in a multi-view scenario. This loss layer first computes the individual log loss of the softmax-normalised output of each Fc8 layer, $\mathcal{V}(i)$ with regard to that of the Fc9 layer, $\mathcal{O}$, which represent the final outputs of the view channel and the object channel respectively. The multi-view loss is then computed as the sum of all individual log losses:

$$
\mathcal{L}=-\sum_{i=1}^{N} \sum_{c=1}^{C} \mathcal{O}_{c} \cdot \log \left(\mathcal{V}_{c}(i)\right)
$$

where for simplicity, we write the output of the view channel $\mathcal{V}_{c}\left(V_{i}\right)$ as $\mathcal{V}_{c}(i)$. Through training, Eq. (7) is minimised by impelling $\mathcal{O}$ to be consistent with $\mathcal{V}(i)$ and the viewobject consistency is thus realised.

It can be clearly seen that the MV-LogLoss defined as Eq. (7) does not rely on any annotations as $\mathcal{O}_{c}$ and $\mathcal{V}_{c}(i)$ are internally generated by the object channel and the view channel of the UMVCNN respectively. $C$ in Eq. (7) is a picked integer defining the output dimension of the Fc8/Fc9 layer when building the UMVCNN. And we shall provide an experimental study on the influence of varying $C$ in Section 4.4.

\subsection{Salient view selection}

In the deployment, given a $3 \mathrm{D}$ object represented as a set of $N$ views, we first feed the views into the UMVCNN and hijack the output of the Softmax layer connected with the Fc9 layer in the object channel during the forward-propagation to predict its object class $\mathcal{C}$. Then, we back-propagate a $C$-dimensional one-hot vector where only the entry of index $\mathcal{C}$ is 1 from this Softmax layer to the input views with all the network weights fixed. This strategy leads to a per-pixel saliency map $I_{i}$ for all the pixels in each view $V_{i}$ based on their influence on the predicted class $\mathcal{C}$. The 2D saliency map $I_{i}$ can be interpreted as a measure of pixel importance with regard to the recognition of the 3D object. Like most methods for salient view selection [16, 24, 17] and also in order to enable a quantitative evaluation, we are keen to evaluate the goodness of any viewpoint on a view sphere, which requires to generate a per-vertex saliency map. To do so, we employed the 2D-to-3D saliency transfer scheme proposed in [26] to derive a 3D saliency map $H_{i}$ from a single 2D saliency map $I_{i}$. Finally, we hijack the output of the VD layer $S_{i}$ as the weighting parameters which represent the learned view distinction to aggregate the multi-view 3D saliency maps $H_{i}$ s into a single one:

$$
H=\sum_{i=1}^{N} S_{i} H_{i} .
$$

We then select the viewpoint that maximises the sum of the saliency map $H$ for the visible regions of the $3 \mathrm{D}$ object as the salient viewpoint:

$$
v_{s}=\arg \max _{v}\left(\sum_{m \in B(v)} H(m)\right)
$$

where $B(v)$ is the set of the vertices visible from the viewpoint $v$ and $H(m)$ denotes the saliency of the vertex $m . M(v)=\sum_{m \in B(v)} H(m)$ can be regarded as the saliency map of the viewpoints. 


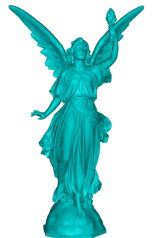

(a)

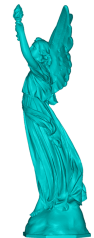

(b)

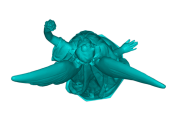

(c)

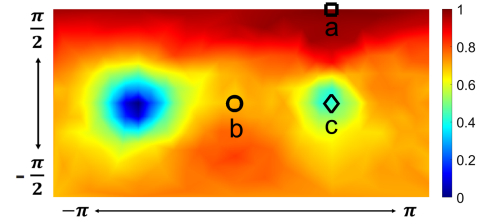

(d)

Fig. 2. Viewpoint saliency map. (a)-(c) are the projected views of the Lucy model. (d) is the viewpoint saliency map where the black square, circle and diamond mark the locations of the viewpoints corresponding to the views shown in (a)-(c) respectively.

Fig. 2 shows the 2D representation of the unwarped viewpoint saliency map on a view sphere normalised to the interval of $[0,1]$. It is generated via the Mercator projection where the $x$ and the $y$ axes correspond to the latitude and the longitude respectively. Note that initially the model is not up oriented in the view sphere.

\subsection{Implementation}

The proposed method is fully unsupervised as it is trained using only a set of 3D objects without any annotations. All we need to do is to pick an integer $C$ for defining the output dimension of $\mathrm{Fc} 8 / \mathrm{Fc} 9$. And the influence of varying $C$ will be studied in Section 4.4.

We first render each 3D object as $202 \mathrm{D}$ views as described in Section 3.1 using a standard OpenGL renderer with perspective projection mode. The strengths of the ambient light, the diffuse light and the specular reflection are set to 0.2, 0.6 and 0.1 respectively. We apply the light uniformly across each triangular face of the meshed object (i.e. flat shading). Note that using different illumination models or shading coefficients does not affect our method due to the invariance of the learned convolutional filters to illumination changes, as observed in image-based CNNs. All of the 20 rendered views are then printed at $200 \mathrm{dpi}$, also in the OpenGL mode, and further resized to the resolution of $224 \times 224$. Then for training we feed these views into the UMVCNN wherein the convolutional layers and the first fully connected layer Fc6 are initialised with the weights pretrained on ImageNet while other fully connected layers Fc7, Fc8 and $\mathrm{Fc} 9$ are all initialised with random weights using the popular method proposed in [11]. The UMVCNN is trained end-to-end through stochastic gradient descent with the learning rate of $10^{-5}$. As we observed, the training always converged within 50 epochs for all of the variants of the UMVCNN that we shall discuss in Sections 4.4. When deploying the UMVCNN to select the salient view of a given 3D object, we again render the object as 20 views with the same rendering settings and then use the scheme described in Sections 3.3 to output the salient viewpoint.

\section{Experimental results}

In this section, we first introduce the datasets used in the experiments and evaluate our method qualitatively. Then, we show that our method can be directly used to select the salient view of a 3D scene to attract further interest. Finally, we evaluate both the proposed UMVCNN and its variants via quantitative comparisons for the demonstration of its superiority as well as a better understanding of our method. 


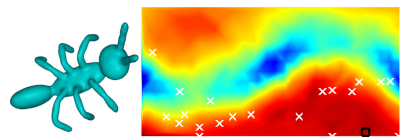

$$
\text { Ant }
$$$$
\text { of }
$$
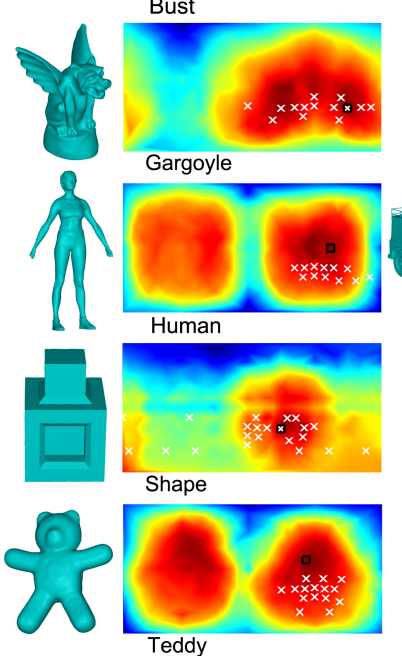
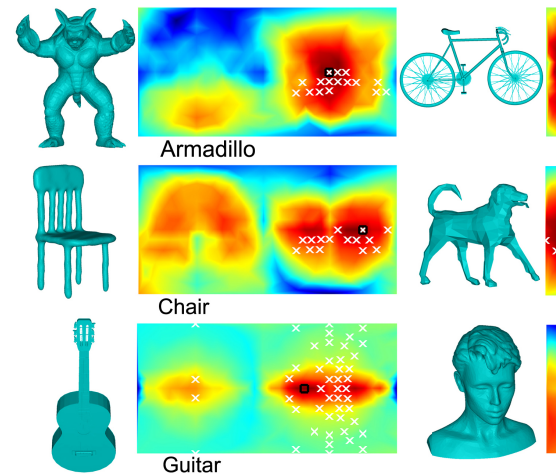

Chair
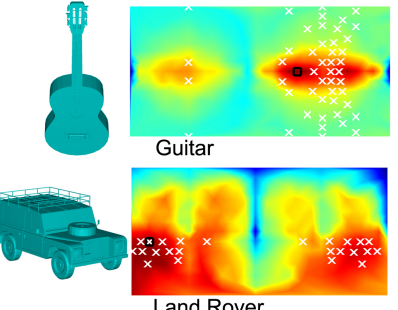

Land Rover
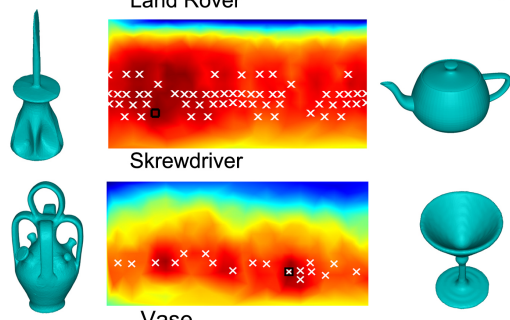
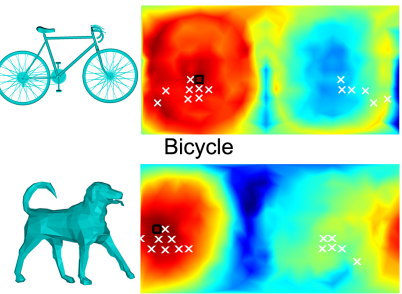

Dog

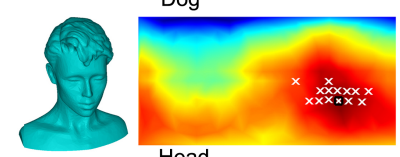

Head

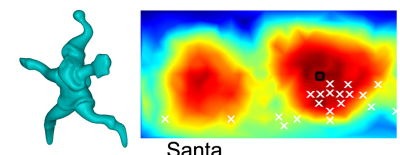

Santa

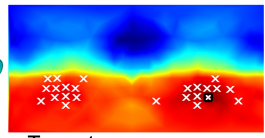

Teapot

Vase

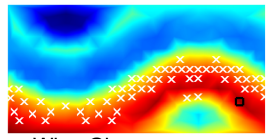

Wine Glass

Fig. 3. Qualitative results of the salient views and the estimated viewpoint saliency maps generated by our method. In each map, the black square corresponds to the salient viewpoints selected by our method. The white "X"s correspond to the ground truth best viewpoints picked by 26 human subjects (including their symmetric viewpoints) via the user study carried out by [7].

\subsection{Datasets}

We create a new dataset containing 2747 3D models downloaded from the Princeton ModelNet dataset [33], the Schelling dataset [4] and the Trimble 3D Warehouse [31]. These models are originally from 30 object categories while in this work, all categorical annotations are removed in training and validation for an unsupervised learning. We use the same data split of ModelNet40 as in [33] where four fifths of the meshes in each category are used for training and one fifth are used for validation.

We test our method on the Best View Selection benchmark [7]. To the best of our knowledge, it is the only one publicly available benchmark suitable for a quantitative evaluation of view selection methods. It contains $683 \mathrm{D}$ objects of various classes and provides a quantitative benchmarking measure, the ground truth best viewpoints picked by 26 people, and the implementation of 7 competing methods. Note that the test dataset contains some objects such as ant, cactus, dragon, fish, octopus, rocker arm, screwdriver, shoe etc. that do not belong to any of the 30 object categories from the perspective of human's object recognition.

We also used the 3D models from the Stanford 3D Scanning Repository [5], the Princeton Shape Benchmark [25] and the Watertight Track of the 2007 Shape-based 

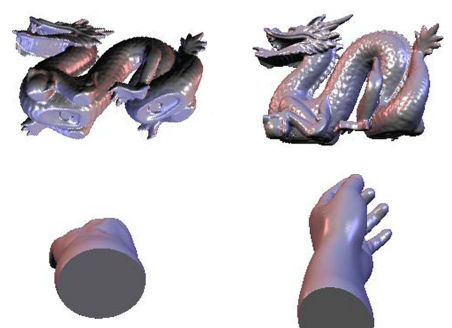

(a)

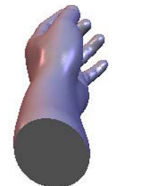

(b)

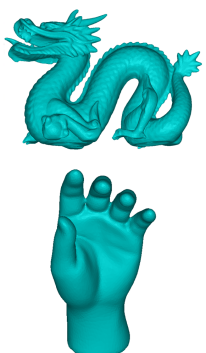

(c)

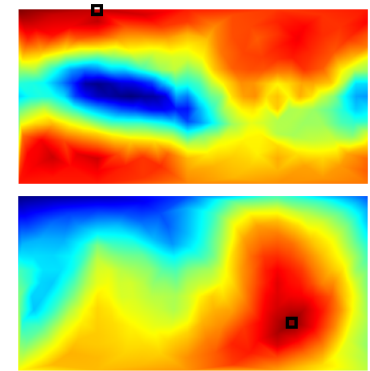

(d)

Fig. 4. Qualitative comparisons with [16] and [34]. (a) The best views selected by [16] (as implemented and shown in [34]). (b) The best views selected by [34]. (c) The best views selected by our method. (d) The viewpoint saliency maps generated by our method where the black squares mark the most salient viewpoints.

Retrieval Contest (SHREC) for qualitative evaluations. We commit to release all the data and codes to the community for public access.

\subsection{Qualitative results}

Fig. 3 shows our results of salient view selection for a variety of $3 \mathrm{D}$ objects, with the ground truth best viewpoints supplied by [7]. It is noteworthy that the ground truth best viewpoints could be more or less than 26 because 1) several human participants could select the same viewpoint and 2) the symmetry of each object is taken into account and thus the symmetric viewpoints of those picked by the participants are also included. It can be seen that the consistency of human preferred viewpoints varies over different objects. Even though, for most objects, the majority of the ground truth best viewpoints fall into the red or orange areas in the viewpoint saliency maps generated by our method, which demonstrates that it is good at predicting human's viewpoint preference over various objects. Also, for most objects, the salient viewpoint found by our method is, or at least very close to, a ground truth viewpoint picked by a human subject. It is worth mentioning that due to the default distortion of the Mercator projection, for the Ant model, the viewpoints on the bottom boundary of the viewpoint saliency map that look distant from each other are actually very close to each other on the view sphere since they are all very close to its bottom pole.

We next compare our results to some produced by competing state-of-the-art methods. Since some of them require tuning of parameters and some are not open-sourced, we used our method to select salient views for the same objects used in the papers where the methods were reported. Fig. 4 compared our method with [16] and [34]. It can be seen that the our method is less influenced by some local geometric features such as the sharp edges at the bottom of the hand model if semantically they do not help the recognition of the object. Similarly, as shown in Fig. 5, the method proposed in [17] chose a back view of the lamp which contains many local details such as wires and screws. In comparison, for both the lamp and the jeep, our method tends to select views natural and good for recognising the objects. Fig. 5 also shows that our method out- 


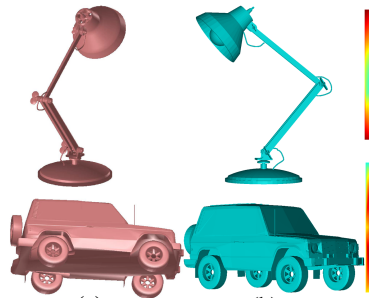

(a)

(b)

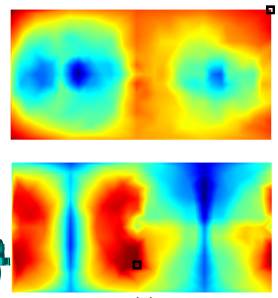

(c)

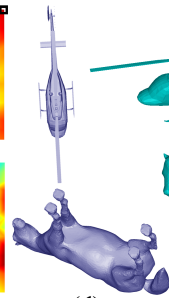

(d)

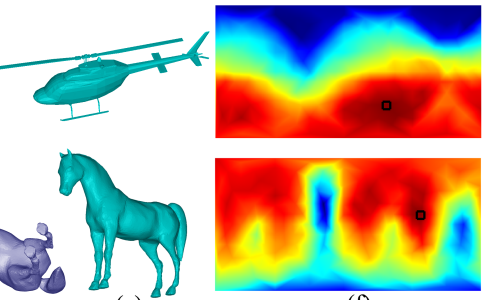

(e)

(f)

Fig. 5. Qualitative comparisons with [17, 26]. (a) and (d) The best views selected by [17] and [26] respectively. (b) and (e) The best views selected by our method. (c) and (f) The viewpoint saliency maps generated by our method where the black squares mark the most salient viewpoints.

performs [26] over a helicopter and a horse model while more convincing quantitative comparisons using a variety of 3D objects are provided in Section 4.3. Note that [26] is essentially based on a weakly supervised deep learning framework where the class labels of the objects are available during training.

Since the UMVCNN does not rely on the knowledge about object classes, our method can be directly used to select the salient view of a 3D scene which usually contains objects of different classes and thus is unlikely to be reliably categorised in most datasets. According to the results shown in Fig. 6, our method successfully selects good views for various 3D scenes. The viewpoint saliency maps of 3D scenes generated by our method are also informative. For instance, by observing the corresponding locations of the best and the worst views in the viewpoint saliency maps of most scenes, we find that the views with positive elevation angles are generally much more salient than those with negative ones, which is consistent with human's viewpoint preference. We also observed that the best view of a scene is not necessarily the best view of each individual object in it. For example, in the living room scene, the best view of the entire scene is not that of one of the three sofas. Similarly, in the work site scene, the best view of the scene is not that of the person in the middle and some chairs.

Please refer to the supplemental material for more qualitative results of salient view selection of 3D objects and scenes.

\subsection{Quantitative results}

We tested our method on the benchmark supplied by [7] which contains 68 objects using a computer with an Intel i7-4790 3.6GHz CPU and 32GB RAM without any GPU acceleration. The salient views of most objects can be computed within 1 minute where the vertex visibility to each viewpoint is precomputed.

Table 1 gives the statistics of the View Selection Error (VSE) of 9 automatic view selection methods over all of 68 objects. The VSE proposed by [7] measures the geodesic distance between the viewpoint found by a method and the ground truth supplied by a human subject on a unit view sphere and is averaged over the choices of all subjects, with the consideration of object-specific symmetry.

According to Table 1, our method yields the best performance in terms of the mean VSE, the median VSE and the number of objects for which a method gave the lowest 

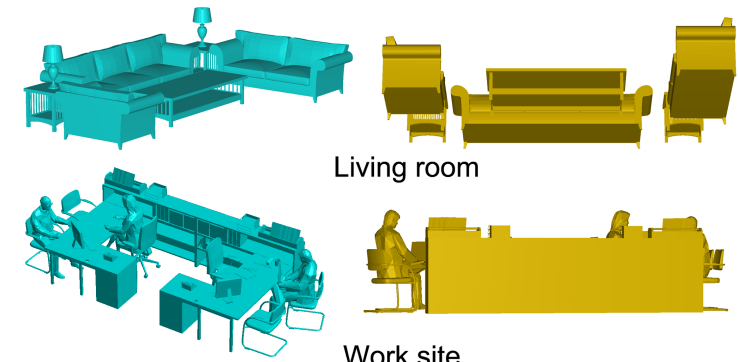

Living room

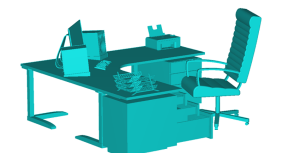

Work site
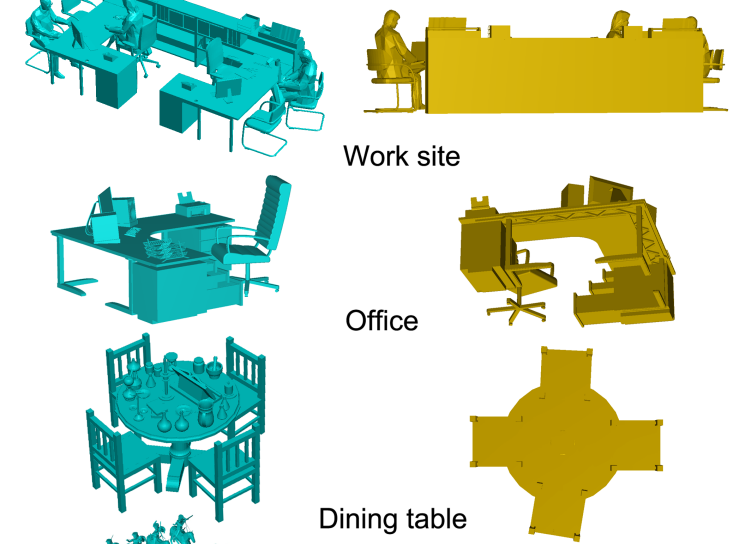

Dining table
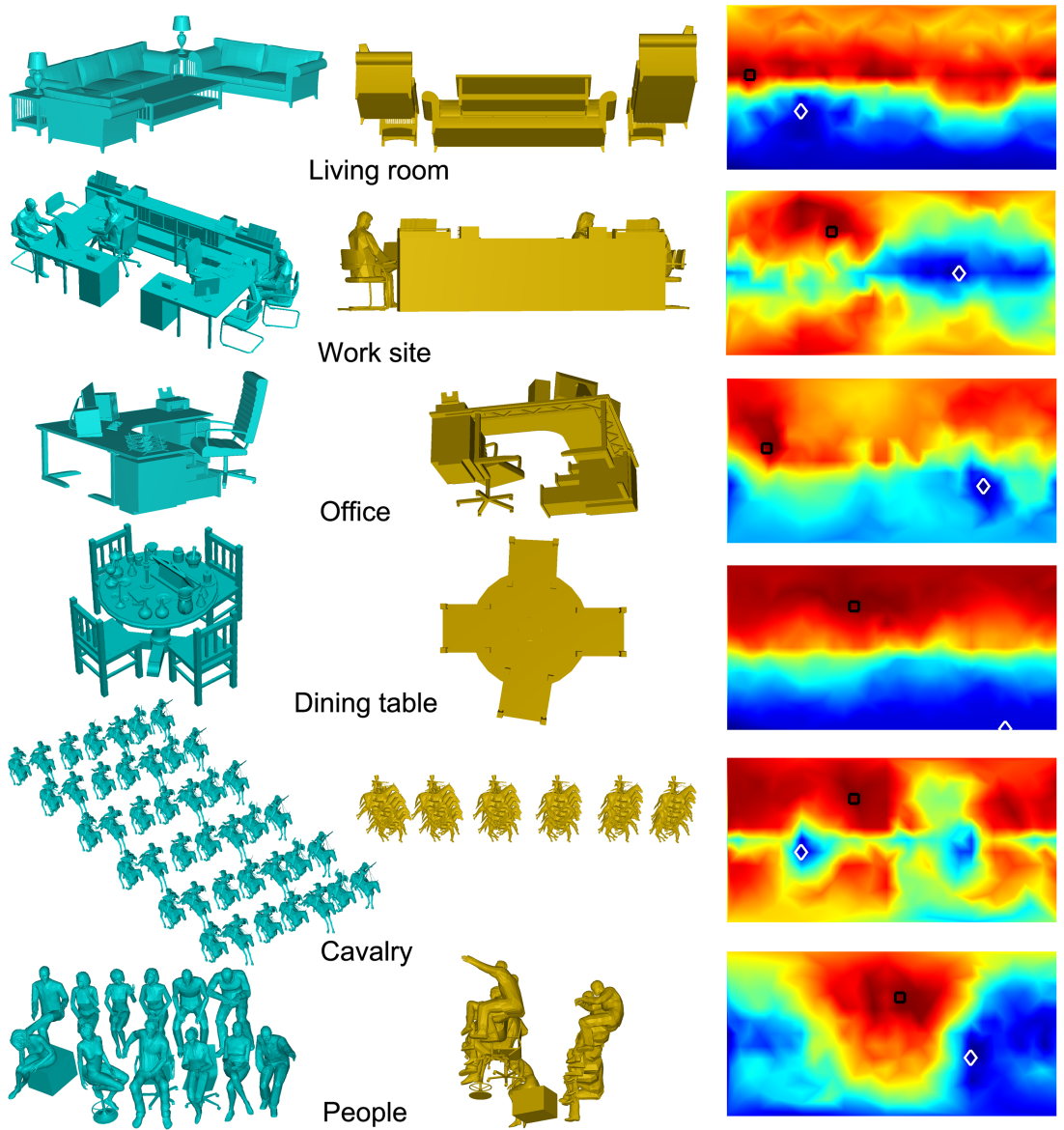

Fig. 6. Salient and non-salient views of 3D scenes (courtesy of the Trimble 3D Warehouse [31]) selected by our method. Left column: the most salient views of the scenes; Middle column: the least salient views of the scenes; Right column: the viewpoint saliency maps of the scenes where the black square marks the most salient view and the white diamond marks the least salient view.

VSE among all competing methods. Here UMVCNN-30 refers to the UMVCNN with $C$ set to 30. As mentioned at the end of Section 3.2, this means that the output dimension of the Fc8 and Fc9 layers is set to 30 when we build the UMVCNN, which indicates that either of the view and the object channels categorises the objects into 30 classes. As shown in Fig. 3, due to the inconsistency of the ground truth choices of human subjects over the same object, reaching a zero mean VSE is impossible and improving the VSE is very challenging if it is already low. In most cases, a viewpoint with a mean VSE lower than 0.3 corresponds to a good view. Even though, our method outperforms the state-ofthe-art method proposed in [26] by 3.4\%, 2.9\%, $4.6 \%$ and $24.8 \%$ in terms of the mean, the median, the standard deviation and the interquartile range of the VSE respectively. Note that their method is also based on deep learning but trained, in a weakly supervised manner, on a large dataset with the annotations of object class membership. 
Table 1. Statistics of the View Selection Error (VSE) of 9 view selection methods over 68 objects. $\mathrm{SD}$ and IQR represent the standard deviation and the interquartile range respectively. $n$ gives the number of objects for which a method gave the lowest VSE among all competing methods.

\begin{tabular}{l|c|c|c|c|c}
\hline \hline View Selection Method & mean VSE & median VSE & SD of VSE & IQR of VSE & $\mathrm{n}$ \\
\hline View area [7] & 0.517 & 0.539 & 0.186 & 0.306 & 6 \\
Ratio of visible area [22] & 0.473 & 0.473 & 0.196 & 0.338 & 1 \\
Surface area entropy [29] & 0.396 & 0.386 & 0.144 & 0.195 & 8 \\
Silhouette length [22] & 0.446 & 0.445 & 0.172 & 0.275 & 7 \\
Silhouette entropy [21] & 0.484 & 0.469 & 0.153 & 0.241 & 5 \\
Curvature entropy [21] & 0.474 & 0.466 & 0.139 & 0.239 & 8 \\
Mesh saliency [16] & 0.430 & 0.395 & 0.165 & 0.233 & 2 \\
Deep mesh distinction [26] & 0.380 & 0.346 & 0.173 & 0.314 & 11 \\
UMVCNN-30 & 0.367 & 0.336 & 0.165 & 0.236 & 20 \\
\hline \hline
\end{tabular}

Table 2. Mean View Selection Error of the variants of the UMVCNN over 68 objects

\begin{tabular}{c|c|c|c|c|c|c|c|c|c}
\hline UMVCNN Variants & $C=10$ & $C=15$ & $C=20$ & $C=25$ & $C=30$ & $\begin{array}{c}C=30, \\
\text { max-pooling }\end{array}$ & $\begin{array}{c}C=30, \\
30 \text { views }\end{array}$ & $C=35$ & $C=40$ \\
\hline mean VSE & 0.379 & 0.373 & 0.382 & 0.381 & 0.367 & 0.384 & 0.366 & 0.377 & 0.380 \\
\hline \hline
\end{tabular}

None of the methods is consistently the best over all the 68 objects although our method accomplishes the best results for 20 objects, the most over all competing methods. This is in agreement with the conclusions in $[1,24]$ which argued that human's view preference is driven by a variety of attributes. But in general, the methods based on low-level attributes perform significantly worse than the two based on deep neural networks which potentially learn some high-level attributes of 3D objects.

In particular, Table 1 shows that our method significantly outperforms [7] based on view area and [22] based on silhouette length in terms of the VSE. This demonstrates that the improvement of the VSE does come from the UMVCNN rather than the handcrafted features, i.e. view area and silhouette length that we use for the multi-view representation of a 3D object (see Section 3.1).

\subsection{Evaluations over the variants of UMVCNN}

Effect of varying $C$. Table 2 gives the mean VSE of the variants of the UMVCNN. We first redesign and test the UMVCNN with different values of $C$. It can be seen that varying $C$ from 30 leads to an insignificant degradation of performance. As mentioned in Section 4.1, the 3D objects used for training are originally from 30 object categories while we removed all categorical annotations in this work for an unsupervised learning. Presumably, that $C=30$ is indeed a good choice for designing the UMVCNN can be interpreted by the fact that salient view selection is a task highly related to $3 \mathrm{D}$ object classification as we observe that the objects of the same class tend to have analogous salient viewpoints while it is not the case the other way round. However, we cannot observe any obvious rule that suggests a way for deciding $C$. In a supervised learning, the network is forced to adopt the taxonomy of object classification consistent with human annotations while there is no guarantee that this taxonomy is optimal to the 

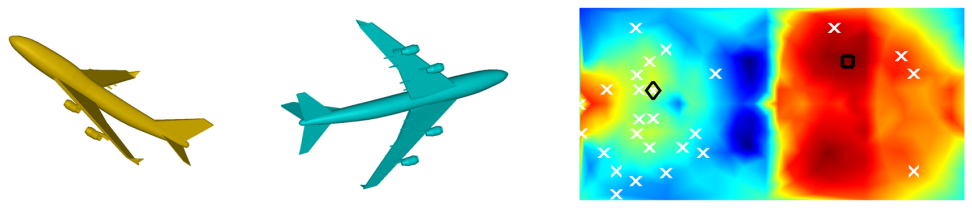

Fig. 7. Limitation. Our method tends to select views good for recognition but not necessarily "natural". Left: the view selected by a subject; Middle: the view selected by our method; Right: the viewpoint saliency map where the diamond and the square mark the views selected by the subject and our method respectively.

particular task such as salient view selection. Thus in different tasks, $C$ might need to

Ablation study for validating VD and WSP. We are also interested in the heuristic component of the UMVCNN, i.e. the VD and the WSP layers of the UMVCNN. To validate its effectiveness, we replace the VD and the WSP layers with the popular element-wise max pooling which have demonstrated state-of-the-art performances in various 3D shape understanding tasks such as classification [27], retrieval [27] and segmentation [14]. The variant corresponds to ' $C=30$, max pooling' in Table 2 . To aggregate the multi-view 3D saliency maps $H_{i}$ in Eq. (8), we set all weighting parameters $S_{i}$ to 1 as it is not available via this variant. As shown in Table 2, the performance of the UMVCNN is significantly worse without the VD and the WSP layers. This demonstrates the effectiveness of the view distinction heuristic we introduced in Section 3.2. It also suggests that the unsupervised learning based on the view-object consistency is likely to benefit from some heuristics introduced for the specific task.

Effect of the number of views. We tested the variant corresponding to ' $C=30$, 30 views' in Table 2 where a 3D object is projected into 30 (i.e. $N=30$ ) instead of 20 views using the method described in Section 3.1. All the other variants in Table 2 used a 20 -view setup. It can be seen that using 30 views merely reduces the mean VSE slightly from 0.367 to 0.366 . Using more or different views is trivial, however, we found that a 20-view setup is already enough to achieve high performance.

\section{Conclusions}

This work reveals that the view-object consistency is promising for the establishment of an unsupervised framework of 3D deep learning. We validate its effectiveness on the challenging task of salient view selection of 3D objects through the relatively naive design of a multi-view deep architecture. While the performance of our method is impressive, it has some limitations as shown in Fig. 7. Our method tends to select a view good for recognising the object, such as the view that better shows some features important for recognising the airplane (e.g. the wings and the engines). However, most human subjects prefer a "natural" side view.

Future work will focus on implementing the unsupervised learning framework in more applications to demonstrate that it is amenable to a wide range of 3D shape understanding tasks. Particularly interesting applications might be some 3D scene understanding tasks hindered by the difficulty of collecting large amounts of accurately and consistently annotated data for training. 
1. Biederman, I.: Recognition-by-components: a theory of human image understanding. Psychological review 94(2), 115 (1987)

2. Blanz, V., Tarr, M.J., Bülthoff, H.H.: What object attributes determine canonical views? Perception 28(5), 575-599 (1999)

3. Chatfield, K., Simonyan, K., Vedaldi, A., Zisserman, A.: Return of the devil in the details: Delving deep into convolutional nets. In: Proc. BMVC (2014)

4. Chen, X., Saparov, A., Pang, B., Funkhouser, T.: Schelling points on 3d surface meshes. ACM Trans. Graph. (Proc. SIGGRAPH) 31(4), 29 (2012)

5. Curless, B., Levoy, M.: A volumetric method for building complex models from range images. In: Proc. SIGGRAPH 1996. pp. 303-312 (1996)

6. Cutzu, F., Edelman, S.: Canonical views in object representation and recognition. Vision Research 34(22), 3037-3056 (1994)

7. Dutagaci, H., Cheung, C.P., Godil, A.: A benchmark for best view selection of $3 \mathrm{~d}$ objects. In: Proc. ACM workshop on 3DOR. pp. 45-50 (2010)

8. Goodfellow, I., Pouget-Abadie, J., Mirza, M., Xu, B., Warde-Farley, D., Ozair, S., Courville, A., Bengio, Y.: Generative adversarial nets. In: Advances in Neural Information Processing Systems. pp. 2672-2680 (2014)

9. Hayward, W.G.: Effects of outline shape in object recognition. Journal of experimental psychology: human perception and Performance 24(2), 427 (1998)

10. He, J., Wang, L., Zhou, W., Zhang, H., Cui, X., Guo, Y.: Viewpoint assessment and recommendation for photographing architectures. IEEE Trans. Vis. Comput. Graph (2018)

11. He, K., Zhang, X., Ren, S., Sun, J.: Delving deep into rectifiers: Surpassing human-level performance on imagenet classification. In: Proc. ICCV. pp. 1026-1034 (2015)

12. Huang, H., Kalogerakis, E., Chaudhuri, S., Ceylan, D., Kim, V.G., Yumer, E.: Learning local shape descriptors from part correspondences with multiview convolutional networks. ACM Trans. Graph. 37(1), 6 (2018)

13. Kalogerakis, E., Averkiou, M., Maji, S., Chaudhuri, S.: 3D shape segmentation with projective convolutional networks. In: Proc. CVPR. vol. 1, p. 8 (2017)

14. Kim, S.h., Tai, Y.W., Lee, J.Y., Park, J., Kweon, I.S.: Category-specific salient view selection via deep convolutional neural networks. In: Computer Graphics Forum. vol. 36, pp. 313-328. Wiley Online Library (2017)

15. Koch, C., Poggio, T.: Predicting the visual world: silence is golden. Nat. Neurosci. 2, 9-10 (1999)

16. Lee, C.H., Varshney, A., Jacobs, D.W.: Mesh saliency. ACM Trans. Graph. (Proc. SIGGRAPH) 24(3), 659-666 (2005)

17. Leifman, G., Shtrom, E., Tal, A.: Surface regions of interest for viewpoint selection. IEEE Trans. Pattern Anal. Mach. Intell 38(12), 2544-2556 (2016)

18. Lienhard, S., Specht, M., Neubert, B., Pauly, M., Müller, P.: Thumbnail galleries for procedural models. In: Computer Graphics Forum. vol. 33, pp. 361-370. Wiley Online Library (2014)

19. Mezuman, E., Weiss, Y.: Learning about canonical views from internet image collections. In: Proc. NIPS. pp. 719-727 (2012)

20. Novotny, D., Larlus, D., Vedaldi, A.: Learning 3d object categories by looking around them. In: Proc. ICCV (Oct 2017)

21. Page, D.L., Koschan, A.F., Sukumar, S.R., Roui-Abidi, B., Abidi, M.A.: Shape analysis algorithm based on information theory. In: Proc. ICIP. vol. 1, pp. I-229 (2003)

22. Polonsky, O., Patané, G., Biasotti, S., Gotsman, C., Spagnuolo, M.: What's in an image? The Visual Computer 21(8-10), 840-847 (2005) 
23. Qi, C.R., Su, H., Nießner, M., Dai, A., Yan, M., Guibas, L.: Volumetric and multi-view cnns for object classification on 3d data. In: Proc. CVPR. pp. 5648-5656 (2016)

24. Secord, A., Lu, J., Finkelstein, A., Singh, M., Nealen, A.: Perceptual models of viewpoint preference. ACM Trans. Graph. 30(5), 109 (2011)

25. Shilane, P., Min, P., Kazhdan, M., Funkhouser, T.: The princeton shape benchmark. In: Proceedings of Shape modeling applications (2004)

26. Song, R., Liu, Y., Rosin, P.L.: Distinction of 3D objects and scenes via classification network and markov random field. IEEE Trans. Vis. Comput. Graph. (2018)

27. Su, H., Maji, S., Kalogerakis, E., Learned-Miller, E.G.: Multi-view convolutional neural networks for 3d shape recognition. In: Proc. ICCV. pp. 945-953 (2015)

28. Tarr, M.J., Pinker, S.: Mental rotation and orientation-dependence in shape recognition. Cognitive psychology 21(2), 233-282 (1989)

29. Vázquez, P.P., Feixas, M., Sbert, M., Heidrich, W.: Viewpoint selection using viewpoint entropy. In: VMV. vol. 1, pp. 273-280 (2001)

30. Vieira, T., Bordignon, A., Peixoto, A., Tavares, G., Lopes, H., Velho, L., Lewiner, T.: Learning good views through intelligent galleries. In: Computer Graphics Forum. vol. 28, pp. 717-726. Wiley Online Library (2009)

31. Warehouse, D.: https://3dwarehouse.sketchup.com

32. Wolfe, J.M.: Guided search 2.0 a revised model of visual search. Psychonomic Bulletin \& Review 1(2), 202-238 (1994)

33. Wu, Z., Song, S., Khosla, A., Yu, F., Zhang, L., Tang, X., Xiao, J.: 3D shapenets: A deep representation for volumetric shapes. In: Proc. CVPR. pp. 1912-1920 (2015)

34. Yamauchi, H., Saleem, W., Yoshizawa, S., Karni, Z., Belyaev, A., Seidel, H.P.: Towards stable and salient multi-view representation of $3 \mathrm{~d}$ shapes. In: IEEE International Conference on Shape Modeling and Applications (2006)

35. Zhao, L., Liang, S., Jia, J., Wei, Y.: Learning best views of $3 \mathrm{~d}$ shapes from sketch contour. The Visual Computer 31(6-8), 765-774 (2015)

36. Zhao, S., Ooi, W.T.: Modeling 3d synthetic view dissimilarity. The Visual Computer 32(4), 429-443 (2016) 